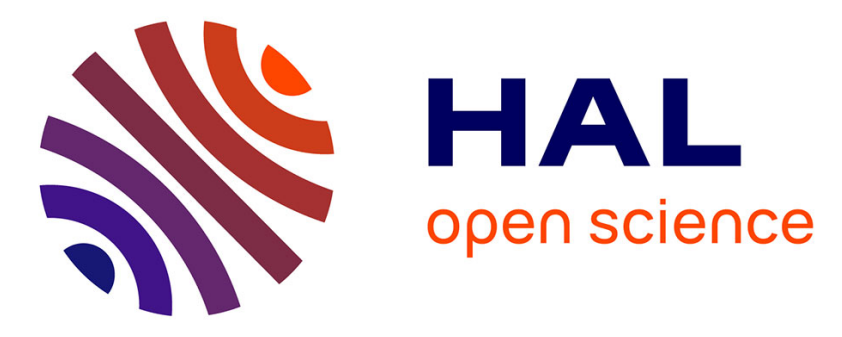

\title{
Effect of hydration and thermal treatment on ceria surface using non-intrusive techniques
}

Jérémy Gaillard, Laurent Venault, Rachel Calvet, Sylvie del Confetto, Nicolas Clavier, Renaud Podor, Michaël Odorico, Jean-Luc Pellequer, Nicolas Vigier, Philippe Moisy

\section{To cite this version:}

Jérémy Gaillard, Laurent Venault, Rachel Calvet, Sylvie del Confetto, Nicolas Clavier, et al.. Effect of hydration and thermal treatment on ceria surface using non-intrusive techniques. Journal of Nuclear Materials, 2014, 444 (1-3), p.359-367. 10.1016/j.jnucmat.2013.10.018 . hal-01625036

\section{HAL Id: hal-01625036 https://hal.science/hal-01625036}

Submitted on 7 Nov 2018

HAL is a multi-disciplinary open access archive for the deposit and dissemination of scientific research documents, whether they are published or not. The documents may come from teaching and research institutions in France or abroad, or from public or private research centers.
L'archive ouverte pluridisciplinaire $\mathbf{H A L}$, est destinée au dépôt et à la diffusion de documents scientifiques de niveau recherche, publiés ou non, émanant des établissements d'enseignement et de recherche français ou étrangers, des laboratoires publics ou privés. 


\title{
Effect of hydration and thermal treatment on ceria surface using non-intrusive techniques
}

\author{
Jérémy Gaillard ${ }^{\mathrm{a}}$, Laurent Venault ${ }^{\mathrm{a}}$, Rachel Calvet ${ }^{\mathrm{b}}$, Sylvie Del Confetto ${ }^{\mathrm{b}}$, Nicolas Clavier ${ }^{\mathrm{c}}$, \\ Renaud Podor ${ }^{\mathrm{c}}$, Michael Odorico ${ }^{\mathrm{d}}$, Jean-Luc Pellequer ${ }^{\mathrm{d}}$, Nicolas Vigier ${ }^{\mathrm{e}}$, Philippe Moisy ${ }^{\mathrm{a}, *}$ \\ a CEA, Nuclear Energy Division, Radiochemistry E Processes Department, BP17171, 30207 Bagnols sur Cèze, France \\ ${ }^{\mathrm{b}}$ Université de Toulouse, Mines Albi, CNRS, Centre RAPSODEE, Campus Jarlard, F-81013 Albi Cedex 09, France \\ 'ICSM-UMR5257 CEA/CNRS/UM2/ENSCM, Site de Marcoule Bat 426, BP17171, 30207 Bagnols sur Cèze, France \\ ${ }^{d}$ CEA, iBEB, Department of Biochemistry and Nuclear Toxicity, F-30207 Bagnols sur Cèze, France \\ e AREVA NC, Boîte à lettre 3448C-1, 1 Place Jean Millier, 92084 Paris La Défense, France
}

\begin{abstract}
A B S T R A C T
The evolution of plutonium dioxide surface due to water adsorption seems to influence $\mathrm{H}_{2}$ generation through the radiolysis of adsorbed water. Surface evolution of ceria, a non-radioactive surrogate for plutonium dioxide, was investigated using Inverse Gas Chromatography (IGC), Raman spectroscopy, Environmental Scanning Electronic Microscopy (ESEM) and Atomic Force Microscopy (AFM). IGC highlights the complexity of ceria surface revealing three different adsorption sites on surface and indicate a surface evolution upon hydration. Thermal treatment appears to regenerate at least partially the initial surface state before hydration. IGC points out the influence of calcination temperature of ceria precursor on surface reactivity. The nature of surface modification was investigated by Raman spectroscopy which suggests formation of superficial hydroxide layer. ESEM and AFM were used to study potential surface topology modification upon superficial layer formation. Cerium hydroxide forms as a superficial layer with a nanostructure differing from the one of the oxide.
\end{abstract}

\section{Introduction}

Plutonium dioxide is one of the end products of the PUREX process dedicated to nuclear fuel recycling. For short term storage, plutonium dioxide, the thermodynamically most stable plutonium phase, is kept in hermetically sealed steel container under atmospheric ambiance [1,2]. The assessment of the radiolytically induced gas generation during plutonium dioxide handling and storage is necessary for transportation and storage risk management [2]. Plutonium dioxide is a hygroscopic material and water can adsorb on the surface to an extent of $2-3$ wt.\% [3,4]. The $\alpha$ radiolysis of the adsorbed water leads to $\mathrm{H}_{2}$ generation [5-9]. The kinetics and extent of $\mathrm{H}_{2}$ generation may depend on the long term surface alteration by water and its radiolysis products. Surface alteration of plutonium dioxide has been investigated using intrusive techniques (microbalance, X-ray photoelectron spectroscopy, UV photoelectron spectroscopy, X-ray diffraction, X-ray absorption fine structure) [3,10-16]. The effect of water adsorption on the surface state is supposed to be minor; it requires to find techniques that modify the least possible reaction equilibria. Inverse Gas Chromatography (IGC), Raman spectroscopy, Environmental Scanning Elec-

\footnotetext{
* Corresponding author. Tel.: +33466791611.

E-mail address: philippe.moisy@cea.fr (P. Moisy).
}

tron Microscopy (ESEM) and Atomic Force Microscopy (AFM) were chosen for their non-intrusive features. Ceria was used as a model compound to investigate the ability of these techniques for the study of surface alteration by hydration. The aim is to study at first nonradioactive materials which enable us to investigate different techniques before implementing these techniques for radioactive materials. Water on oxide surfaces is both chemisorbed and physisorbed. The first layer is strongly bound to the surface (chemisorption). The first layer might be responsible for surface evolution because chemisorption leads to chemical bound between hydroxyl group and the surface $[17,18]$. On plutonium oxide surface water adsorbs in three different stages: chemisorption, quasi-chemisorption (hydrogen bound to the first layer), physisorption [3]. The chemisorbed water requires very high temperature superior to $800{ }^{\circ} \mathrm{C}$ for desorption showing very strong bonding [3]. Water adsorption on ceria surface is a controversial subject since water seems to promote oxidation of non-stoichiometric surface and reduction of the stoichiometric surface depending on orientation and surface structure [19]. Calorimetric measurements allows to highlight two different adsorption sites for water on the surface corresponding to a structural cerium(IV) site and oxygen vacancy site [20]. Water molecule dissociation is controversial, however dissociation looks to be promoted by surface oxygen vacancies [21]. DFT simulation of water adsorption on low index ceria surface shows that water dissociation is 
thermodynamically more favorable on reduced ceria surface [19]. The presence of oxygen vacancy increases the adsorption energy which is favorable to dissociation. Indeed the results show that substoichiometric surfaces are stabilized by dissociative water adsorption. The behavior of water molecules on $\mathrm{PuO}_{2}$ surface is not known as well as on $\mathrm{CeO}_{2}$ surface, however $\mathrm{PuO}_{2}$ surface is expected to have a similar behavior to $\mathrm{CeO}_{2}$.

In the present study, IGC was used to study ceria surface evolution during atmospheric hydration. IGC is a physico-chemical method dedicated to surface analysis which is based on surface energy measurement. The use of molecular probes permits non-intrusive measurement ensuring an efficient surface state investigation. Both Infinite dilution method (IGC-ID) and finite solute concentration method (IGC-FC with elution characteristic point method) are applied. The finite solute concentration method was used to map surface adsorption site and especially to evaluate the surface energetic heterogeneity [22]. The infinite dilution method allowed to monitor surface evolution with hydration and thermal treatment. Although IGC is widely used for polymer surface characterization [23], inorganic materials have been studied like oxides [22,24,25], clays [26] or zeolites [27].

Raman spectroscopy was used to study evolution of the vibrational behavior of the ceria surface induced by hydration. Raman spectroscopy has already been applied to actinide oxides as $\mathrm{ThO}_{2}$, $\mathrm{UO}_{2}, \mathrm{NpO}_{2}$ and $\mathrm{PuO}_{2}$ [28-34]. Raman spectra of $\mathrm{CeO}_{2}$ show one major vibration band corresponding to $\mathrm{T}_{2 \mathrm{~g}}$ mode at $465 \mathrm{~cm}^{-1}$. Additional bands corresponding to $1 \mathrm{LO}$ and $2 \mathrm{LO}$ mode can be observed at $595 \mathrm{~cm}^{-1}$ and $1180 \mathrm{~cm}^{-1}$ [35]. The first order LO band scattering modes are normally forbidden in the fluorite structure. Disorders in the crystal lattice due to chemical change can lead to the apparition of these bands. Recently, the existence of $1 \mathrm{LO}$ band on $\mathrm{PuO}_{2}$ spectrum has been assigned to lattice defects due to radiation damage [34]. No characteristic band is assigned to surface alteration by hydration. In the case of $\mathrm{PuO}_{2}$, additional bands are observed at 2116 and $2611 \mathrm{~cm}^{-1}$ which seem to have electronic origin [34]. A potential evolution of material might be induced by laser beam [28] and experimental method is adapted in order to insure non-intrusive measurements.

ESEM allows to follow potential evolution of the microstructure of the oxide. ESEM enables to study surfaces in higher pressure than conventional scanning electronic microscope (SEM) and to hydrate sample in situ. Reaction equilibria are then less modified compared to ultra-high vacuum conditions. Indeed ultra-high vacuum conditions are too intrusive to ensure the preservation of the tenuous equilibrium of hydration of the surface. ESEM using much higher pressure is equipped with a Peltier setup. It permited to control the relative humidity in the ESEM to hydrate samples in situ by cooling down humid atmosphere and enables to examine potential evolution of the microstructure during hydration. AFM was applied for its capacity to discern surface topology modification at a nanometer scale [36]. AFM enables to study the surface topology at atmospheric pressure which prevents any surface evolution induced by low pressure environment. AFM is usually used with oxide thin films $[37,38]$ but in our study, the preparation route precludes the formation of thin films. AFM was then applied directly on powder particles scattered on mica support.

The objective is to study the hydration of ceria surface with independent methods (IGC, Raman spectroscopy, ESEM and AFM) helping to validate these techniques for implementing on radioactive materials. Ceria is furthermore a classic surrogate for plutonium dioxide [39]. Oxides were prepared using the same procedure used for plutonium oxide in the PUREX process, that is to say oxalate calcination. Oxalate as precursor leads to oxide with particular features (specific surface area, porosity) [40]. Cerium oxide used in this study has characteristic close to $\mathrm{PuO}_{2}$ prepared with the same oxalate route.

\section{Experimental section}

All chemicals were pure grade and supplied by Sigma Aldrich. Oxalate of $\mathrm{Ce}(\mathrm{III})$ were precipitated from cerium nitrate solution adding oxalic acid. The nitrate solution $(0.25 \mathrm{M})$ was stirred using magnetic stirrer while adding $0.525 \mathrm{M}$ oxalic acid. Precipitation was performed under ambient temperature. The solution was filtered; the filtrate was washed with $1 \mathrm{M}$ nitric acid solution and finally washed with a mixture of ethanol/water (50/50). A first precipitation batch (batch A) led to an oxalate powder which is calcined under air at two different temperatures, $650^{\circ} \mathrm{C}$ and $800^{\circ} \mathrm{C}$, to form $\mathrm{CeO}_{2}$ (mass(oxide) $=9.135 \mathrm{~g}$, Table 1 ). The temperature ramp was $10^{\circ} \mathrm{C} \mathrm{min}^{-1}$ and calcination temperature was hold $5 \mathrm{~h}$. The temperature of $650{ }^{\circ} \mathrm{C}$ and $800{ }^{\circ} \mathrm{C}$ were chosen to obtain an important difference of specific surface area. Calcination at $650{ }^{\circ} \mathrm{C}$ leads to an oxide with a specific surface area at least two times higher than calcination at $800{ }^{\circ} \mathrm{C}[40,41]$. The batch $\mathrm{A}$ was dedicated to the study of surface hydration and thermal treatment by IGC. These oxides compounds were characterized using X-ray diffraction and BET specific surface area measurement. A part of the $\mathrm{CeO}_{2}$ powder was stored at room temperature under non-specific conditions (reference samples). Two samples (differenced by calcination temperatures) were stored in a closed vessel in order to be contacted with humid atmosphere at $80 \%$ (hydrated_1 and hydrated_2). Two hydrated samples were taken from the closed vessel and underwent thermal treatment after hydration (treated_1 and treated_2). Samples were treated at $550^{\circ} \mathrm{C}$ during $3 \mathrm{~h}$ under argon and $3 \mathrm{~h}$ under air. Temperature was kept $100^{\circ} \mathrm{C}$ below calcination temperature. This parameter prevents reduction of surface area. A last stage was performed into air to ensure stoichiometry of the surface known to influence surface reactivity [42]. These samples (treated_1 and treated_2) were stored before analysis in tightly closed vessels to prevent evolution of the surface. These samples are referenced $\mathrm{A}$ in the Table 1 . For general characterizations (Raman spectroscopy, ESEM, AFM), a second batch B of cerium oxalate was synthesized using the same procedure and calcined under air at $800{ }^{\circ} \mathrm{C}($ mass $($ oxide $)=39.854 \mathrm{~g})$.

Besides characterization of ceria surface, cerium hydroxide were also characterized by Raman spectroscopy. Cerium(III) and cerium(IV) hydroxide were precipitated from a nitrate cerium(III) and a sulfate cerium(IV) solution by bubbling $\mathrm{NH}_{3}$. The $\mathrm{NH}_{3}$ flow stream was set up by bubbling argon in a concentrated ammoniac solution (27\%).

The specific surface area was measured by the BET method with a Micromeretics Gemini 2360 apparatus. The surface area of ceria samples before hydration or thermal treatment is shown in Table 1. It should be noted that this method requires thermal treatment of the sample at $350{ }^{\circ} \mathrm{C}$ during $4 \mathrm{~h}$. These low specific surface areas are related to the preparation method and calcination temperatures. The decrease of specific surface areas with the increase of calcination temperature is due to pores disappearance $[40,42]$. Xray powder diffraction (with gold as standard) experiments were performed with a Bruker AXS D8 diffractometer (curved positionsensitive detector) using $\mathrm{Cu}$ radiation $(\mathrm{K} \alpha 1=1.5406 \AA$, $\mathrm{K} \alpha 2=1.5444 \AA$ ) equipped with a LynxEye angular detector. Lattice parameters were calculated using FULLPROF software with Le Bail - full pattern matching method. Results are shown in Table 1. The diffraction patterns are in good agreement with literature showing the characteristic lines of the fluorite structure (space group $\mathrm{Fm} \overline{3} \mathrm{~m}$ ). XRD patterns on reference $\mathrm{CeO}_{2}$ and hydrated $\mathrm{CeO}_{2}$ showed the classic pattern for $\mathrm{CeO}_{2}$ in both cases. The lattice parameter for both samples is consistent with literature [43]. The lattice parameter is not changed over hydration of the sample. Thus, the crystalline structure of the bulk oxide is not modified during the hydration as it can be expected since X-ray penetrates at a depth (several $\mu \mathrm{m}$ ) where hydration is not supposed to occur. 
Table 1

Ceria samples preparation.

\begin{tabular}{|c|c|c|c|c|c|c|}
\hline Batch & Sample & $\begin{array}{l}\text { Calcination } \\
\text { temperature }\end{array}$ & $\begin{array}{l}\text { Hydration (relative humidity/ } \\
\text { duration) }\end{array}$ & $\begin{array}{l}\text { Thermal treatment } \\
\text { (temperature) }\end{array}$ & $\begin{array}{l}\text { Surface area }\left(\mathrm{m}^{2} /\right. \\
\mathrm{g})\end{array}$ & $\begin{array}{l}\text { Lattice parameter } \\
(\AA)\end{array}$ \\
\hline \multirow[t]{6}{*}{ A } & Reference_1 & $800^{\circ} \mathrm{C}$ & - & - & $4.3 \pm 0.2$ & $5.4106(5)$ \\
\hline & Reference_2 & $650^{\circ} \mathrm{C}$ & - & - & $9.5 \pm 0.5$ & $5.4104(1)$ \\
\hline & Hydrated_1 & $800^{\circ} \mathrm{C}$ & $80 \% / 16$ days & - & ${ }_{-}^{a}$ & \\
\hline & Hydrated_2 & $650^{\circ} \mathrm{C}$ & $80 \% / 16$ days & - & $-{ }^{a}$ & \\
\hline & Treated_1 & $800^{\circ} \mathrm{C}$ & $80 \% / 16$ days & $550^{\circ} \mathrm{C}$ & $--^{a}$ & \\
\hline & Treated_2 & $650^{\circ} \mathrm{C}$ & $80 \% / 16$ days & $550{ }^{\circ} \mathrm{C}$ & $-^{\mathrm{a}}$ & \\
\hline B & - & $800^{\circ} \mathrm{C}$ & - & - & $3.4 \pm 0.2$ & $5.4109(1)$ \\
\hline
\end{tabular}

${ }^{a}$ BET measurement requires thermal treatment which might modify surface state.

Raman experiments were performed on a Horiba-Jobin Yvon Aramis apparatus equipped with an edge filter and using a green YAG $(\lambda=532 \mathrm{~nm})$ laser. The laser beam was focused on the sample using an Olympus BX 41 microscope, resulting in a spot area of about $1 \mu \mathrm{m}^{2}$. Spectra were recorded from 100 to $900 \mathrm{~cm}^{-1}$. Three different locations were investigated at the surface of each sample, considering a dwell time of $60 \mathrm{~s}$ and 3 scans. $\mu$-Raman bands areas were then determined from a Gaussian fitting using the Origin 6 software. The Raman spectroscope is equipped with an LINRAMT-1500 heating device with a quartz window enabling measurement under high temperature.

SEM observations were performed with a FEI Quanta 200 environmental scanning electron microscope (ESEM) using a field emission gun. ESEM is equipped with a Peltier device enabling in situ hydration.

AFM experiment were performed with a commercial AFM, Multimode and Nanoscope V controller (Veeco, Santa Barbara, CA), mounted with an EV Scanner and antimony $(n)$ doped silicium tips $(K=5 \mathrm{~N} / \mathrm{nm}$, Bruker). Amplitude images were recorded in tapping mode in air at the cantilever resonance frequency. The resulting images were plane-fitted and flattened with the supplied Nanoscope analysis software (Bruker).

The IGC chromatographic experiments were carried out with two Agilent 6890 gas chromatographs equipped with two flame ionization detectors (FID). Helium was used as carrier gas with a flow rate of about $30 \mathrm{~cm}^{3} \mathrm{~min}^{-1}$ measured with electronic flowmeter. The injector and detector temperatures were set at $170^{\circ} \mathrm{C}$. Columns were filled with ceria in amount limiting pressure drop under 2 bars. Columns were packed using mechanical vibration along column. Ends were plugged with glass beads (a non-interacting material). Columns characteristics are described in Table 2. Columns were conditioned at $250{ }^{\circ} \mathrm{C}$ overnight. At this temperature, only physisorbed water is desorbed. This latter is not expected to interfere with surface evolution (especially for hydrated and treated materials). The chromatograms were treated with the software supplied by Adscientis (France). Each experiment was repeated three times in order to determine the reproducibility of the measurement. Probe molecules were prepared from Sigma Aldrich analytical grade products (pentane, hexane, heptane, octane and

Table 2

IGC column characteristics.

\begin{tabular}{llllll}
\hline Batch & Sample & $\begin{array}{l}\text { Length } \\
(\mathrm{cm})\end{array}$ & $\begin{array}{l}\text { Diameter } \\
(\mathrm{mm})\end{array}$ & $\begin{array}{l}\Delta P \\
(\mathrm{mbar})\end{array}$ & $\begin{array}{l}\text { Mass } \\
(\mathrm{g})\end{array}$ \\
\hline A & Reference_1 & 10 & 6.35 & 0.815 & 2.193 \\
& Reference_2 & 10 & 3.17 & 1.521 & 0.497 \\
& Hydrated_1 & 10 & 3.17 & 1.766 & 0.620 \\
& Hydrated_2 & 10 & 3.17 & 1.165 & 0.476 \\
& Treated_1 & 10 & 3.17 & 1.838 & 0.617 \\
& Treated_2 & 10 & 3.17 & 1.622 & 0.506 \\
B & IGC-FC & 10 & 6.35 & 1.253 & 2.940 \\
\hline
\end{tabular}

$\Delta P$ represents pressure drop at $25^{\circ} \mathrm{C}$. isopropanol). Methane was bubbled in liquid sample of probe before experiments for chromatographic reference.

\section{IGC measurements}

IGC is based on the elution of molecular probes on chromatographic column packed with the under-study material. Unlike the conventional gas chromatography dealing with gas separation, IGC permits characterization of the stationary phase. The elution of specific probes molecules allows to obtain useful information on the surface state. Depending on the amount of injected probe, two types of IGC may be distinguished: infinite dilution (IGC-ID) or finite solute concentration (IGC-FC).

\subsection{Evaluation of surface heterogeneity}

The finite solute concentration method (IGC-FC) is an experimental method of IGC based on the determination of desorption isotherm by inverse gas chromatography [44]. Unlike the infinite dilution method, a large amount of probe molecules is introduced and eluted on the column in order to adsorb one theoretical monolayer of probe molecules on surface leading to a strong asymmetric peak. Since all the surface is covered by probe molecules, this method allows to obtain information about all kinds of adsorption sites on the surface. Experimentally, the oven temperature was set to $43^{\circ} \mathrm{C}$ ensuring a slow desorption of the probe molecules. An other criterion for oven temperature is that the contribution of the probe desorption to the total flow rate must be less than $5 \%$ of the flow rate in the absence of probe molecules (Conder criterion). This condition allows to assume perfect gas behavior of probe molecule vapor and to calculate partial pressure. When the signal is back to baseline, an increase of the oven temperature to $250{ }^{\circ} \mathrm{C}$ was applied to evacuate the irreversibly adsorbed molecules on surface at $43^{\circ} \mathrm{C}$.

The desorption isotherm is calculated from the chromatographic peak shape which is related to partial pressure of probe and surface coverage. In fact, the first derivative of the isotherm is related to the net retention time at each point of the chromatographic peak (Eq. (1)):

$\left(\frac{d N_{a d s}}{d P}\right)_{L, t_{r}}=D_{c} \frac{\left(t_{r}-t_{0}\right)}{m R T}$

where for a given characteristic point, $N_{a d s}$ is the number of adsorbed probe molecules, $P$ is the partial pressure of the probe at the output of the column - directly related to the height of the signal, $t_{r}$ is the retention time and $t_{0}$ the retention time of $\mathrm{CH}_{4}-\mathrm{a}$ molecule not retained by the solid surface, $D_{c}$ is the corrected flow rate, $\mathrm{m}$ is the mass of the solid contained in the column.

Since the shape of the chromatographic peak is related to the interaction of the molecular probe with the surface, the isotherm includes information about the surface heterogeneity. 
The distribution function is calculated as described by Balard et al. [45] using Rudzinski-Jagiello approximation based on a model assuming that the global isotherm is a sum of local isotherms corresponding to isoenergetic domains. Then the distribution function is related to the proportion of each domain on the surface.

The distribution function is calculated using the integral (Eq. (2)):

$N\left(P_{m}, T_{m}\right)=N_{0} \int_{\varepsilon_{\min }}^{\varepsilon_{\max }} \theta\left(\varepsilon, P_{m}, T_{m}\right) \chi(\varepsilon) d \varepsilon$

where $N\left(P_{m}, T_{m}\right)$ is the number of molecules adsorbed at the pressure $P_{m}$ and temperature $T_{m}$ of measurement, $N_{0}$ is the number of molecules needed for the formation of a monolayer, $\theta\left(\varepsilon, P_{m}, T_{m}\right)$ is the local isotherm, $\varepsilon$ is the adsorption energy of a site, and $\chi(\varepsilon)$ is the distribution function (DF) of the sites seen by the probe. The range of adsorption energies is included between minimal $\left(\varepsilon_{\min }\right)$ and maximal $\left(\varepsilon_{\max }\right)$ values.

\subsection{Surface monitoring by $\gamma_{\mathrm{s}}^{\mathrm{d}}$ measurement}

Surface energy is divided in two components: a dispersive component $\gamma_{s}^{\mathrm{d}}$ and a specific component $\gamma_{\mathrm{s}}^{\mathrm{sp}}$. The first one is related to London interactions between molecules and surface while the specific component is related to specific interactions (acid-base interactions, polar interactions, hydrogen bond, etc.). These two parameters are determined by IGC-ID respectively by injection of linear alkanes and specific probes. In this paper, only the determination of the dispersive component will be described.

The infinite dilution method (IGC-ID) consists in eluting very low amount of molecular probes [44]. It prevents interactions between probe molecules in the gas phase or on the surface. Experimentally, the oven temperature was set to $150^{\circ} \mathrm{C}$. The obtained chromatogram reflects only interactions between an isolated probe molecule and the surface. This method is based on the measurement of the net retention volume $V_{n}$ of a specific probe which is related to the interaction between the molecular probe and the surface [44]. The net retention volume leads to the calculation of the free adsorption energy $\Delta D_{a}^{0}$ (Eq. (3)).

$\Delta G_{a}^{0}=-R T \ln \left(V_{n}\right)+C$

where $C$ is a constant depending on the arbitrary choice of the reference state of the adsorbed molecule.

The net retention volume is calculated using a corrected flow rate of the carrier gas to take into account the drop pressure induced by the column.

Experimentally, the elution of a set of n-alkanes allows to plot the free adsorption energy versus the number of carbon atoms of the alkane molecule. The linear plot obtained is called the n-alkane line whose slope corresponds to the variation of the free adsorption energy of one methylene group $\Delta G_{a}^{\mathrm{CH}_{2}}$. n-alkanes have the property of developing only London interactions (as known as dispersive interactions) with the surface. Using $\Delta G_{a}^{\mathrm{CH}_{2}}$, one can readily calculate the value of the dispersive component of surface energy [46]. IGC-ID helps to follow surface evolution using the variation of dispersive component $\gamma_{s}^{\mathrm{d}}$.

$\gamma_{\mathrm{s}}^{\mathrm{d}}=\frac{\left(\Delta G_{a}^{\mathrm{CH}_{2}}\right)^{2}}{4 N_{a}^{2} a_{\mathrm{CH}_{2}}^{2} \gamma_{\mathrm{CH}_{2}}}$

where $N_{a}$ is Avogadro's number, $a_{\mathrm{CH}_{2}}$ is the estimated area of one adsorbed methylene group $\left(0.06 \mathrm{~nm}^{2}\right)$, and $\gamma_{\mathrm{CH}_{2}}$ is the surface energy of pure methylene group surface, i.e., polyethylene, $\gamma_{\mathrm{CH}_{2}}=35.6+0.058(293-T)$, in $\mathrm{mJ} / \mathrm{m}^{2}(T$ the temperature in $\mathrm{K})$ [24].
Since a very low amount of probes is eluted, only the most energetic adsorption sites mainly influence the retention times. Thus the result reflects the behavior of these particular adsorption sites which are used to testify for surface evolution. This method is chosen for its versatility compared to the finite solute concentration method for study evolution upon hydration.

\section{Results and discussion}

\subsection{Evaluation of surface heterogeneity by finite solute concentration method}

IGC-FC method was applied to identify different adsorption sites on ceria sample calcined at $800^{\circ} \mathrm{C}$. Isopropanol appeared to have a suitable behavior for investigating ceria surface, it means respect of Conder criterion and an adsorption mainly reversible at $43{ }^{\circ} \mathrm{C}$. Alkanes were excluded because the Conder condition was not met. 1,4-Dioxane adsorption was mainly irreversible on ceria surface at low temperature. Once the suitable probe selected, one must find the probe amount to inject corresponding to one monolayer. Here, injected volume was $2.05 \mu \mathrm{L}$. Experimentally, increasing quantities of probes are injected, the diffuse fronts of the chromatographic peaks are recovering themselves (see Fig. A.1, supporting information). The top of the peaks appears for lower and lower retention times until a minimum. If the injected amount is still increased, distorted peak shapes are obtained suggesting a multilayer adsorption (see Fig. A.1, supporting information).

From the chromatogram, the desorption isotherm was calculated using Eq. (1) (see Fig. A.2, supporting information) and the adsorption energy distribution function was deduced using Eq. (2). The calculated adsorption energy distribution function of isopropanol on ceria surface at $43{ }^{\circ} \mathrm{C}$ is presented in the Fig. 1. Curve fitting suggests three different kinds of adsorption sites as three Gaussian peaks are needed to fit the curve. The fitting parameters are described in Table 3. Fitting criterion is to require a minimum number of Gaussian peaks to properly fit the experimental curve. The first Gaussian peak is centered on $17.4 \pm 0.01 \mathrm{~kJ} \mathrm{~mol}^{-1}$. This peak corresponds to the low energy sites. The surface of the first Gaussian peak strongly suggests that the low adsorption energy sites represent the majority of the adsorption sites on the surface. Two other peaks appear at $23.5 \pm 0.07$ and $27.4 \pm 0.08 \mathrm{~kJ} \mathrm{~mol}^{-1}$. These two peaks are related to high adsorption energy sites. The fitting curve is not in good agreement with the experimental curve at low (under $12.5 \mathrm{~kJ} \mathrm{~mol}^{-1}$ ) and high (over $30 \mathrm{~kJ} \mathrm{~mol}^{-1}$ ) energy. Low energy adsorption sites correspond to the first probe molecules eluted from the surface, i.e. the top of the chromatographic

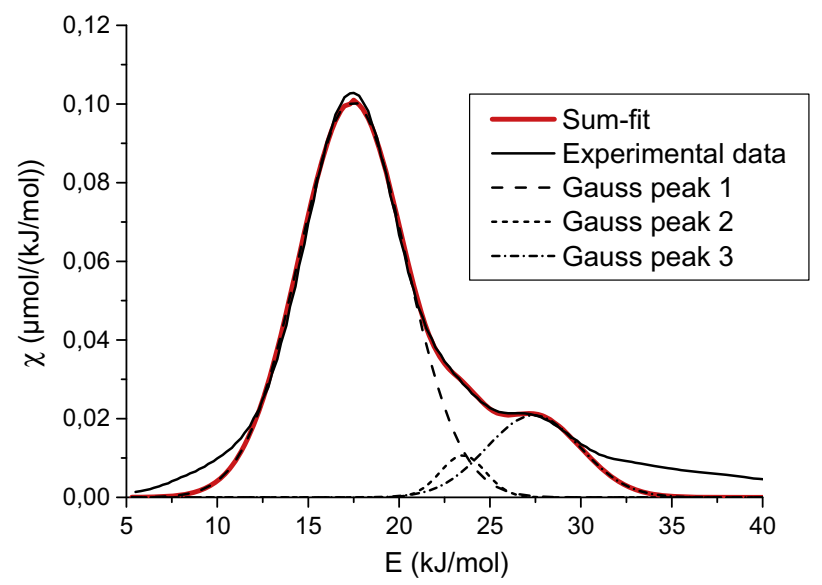

Fig. 1. Energy site distribution function in Rudzinsky-Jagiello approximation. 
Table 3

Fit parameters for adsorption energy distribution function.

\begin{tabular}{lll}
\hline Peak & Center $\left(\mathrm{kJ} \mathrm{mol}^{-1}\right)$ & Area \\
\hline Gaussian peak 1 & $17.4 \pm 0.01$ & 0.742 \\
Gaussian peak 2 & $23.5 \pm 0.07$ & 0.033 \\
Gaussian peak 3 & $27.4 \pm 0.08$ & 0.135 \\
\hline
\end{tabular}

peak. The signal recorded by gas chromatograph is then very high and sensibility of detector is altered. High energy adsorption sites refer to the last molecules eluted and the signal is very close to baseline and the noise to signal ratio is high. In these conditions, the calculation of the adsorption energy distribution function is less reliable.

Ceria surface appears to be a complex surface with a high surface heterogeneity including three different adsorption sites. The complex conversion to oxide from oxalate likely explains the complexity of the surface.

\subsection{Surface characterization by $\gamma_{s}^{\mathrm{d}}$ measurement}

In this study, methane was used as a non-interacting reference and pentane, hexane, heptane and octane as molecular probes. The results obtained with two reference samples are shown in Fig. 2 and Table 3. Probes were collected from partial pressure in equilibrium with liquid phase alkanes. Progressively lowered amount of probes were injected until retention time became constant and peak symmetric. Examples of n-alkanes lines are given in supporting information (Fig. A.3, supporting information).

The dispersive component of the surface energy $\gamma_{s}^{\mathrm{d}}$ decreases from $221 \pm 2 \mathrm{~mJ} \mathrm{~m}^{-2}$ to $184 \pm 2 \mathrm{~mJ} \mathrm{~m}^{-2}$ with the increase of the oxalate calcination temperature. Increasing calcination temperature induces the stabilization of the surface regarding molecular probes adsorption, i.e. the surface is less reactive towards molecular probes adsorption as calcination temperature increases. The comparison of $\gamma_{s}^{\mathrm{d}}$ values with literature $[24,27]$ shows that the surface is very energetic close to activated carbon and zeolite $5 \mathrm{~A}$ (Table 4). A previous study on ceria by Dìaz et al. gives a much lower value $\left(27.5 \mathrm{~mJ} \mathrm{~m}^{-2}\right)$ for the dispersive component of the surface energy of ceria [24]. This observation might be related to preparation method which is different from the present study. The preparation of ceria is not described by Dìaz et al. IGC columns are filled by crushed pellets about $250-350 \mu \mathrm{m}$ in size. Here, samples were fine powder (granulometry $\sim 5 \mu \mathrm{m}$ [24]) leading to a packed column. The decrease of the particle size is already known in some cases for increasing the $\gamma_{s}^{\mathrm{d}}$ value [47] due to the appearance of

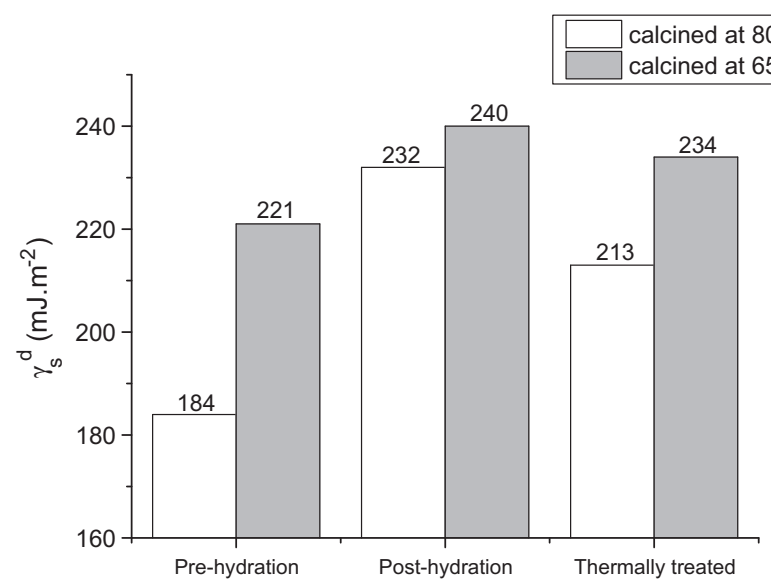

Fig. 2. $\gamma_{s}^{\mathrm{d}}$ measurements on ceria surface with different treatment and different calcination temperature (light grey: $650^{\circ} \mathrm{C}$; dark grey: $800^{\circ} \mathrm{C}$ ), uncertainty: \pm 2 .
Table 4

$\gamma_{\mathrm{s}}^{\mathrm{d}}$ values for different inorganic materials for reference and (pre-hydration) ceria samples.

\begin{tabular}{llll}
\hline Materials & Preparation & $\begin{array}{l}\gamma_{s}^{\mathrm{d}} \\
\left(\mathrm{mJ} \mathrm{m}^{-2}\right)\end{array}$ & Reference \\
\hline $\begin{array}{l}\text { Alumina } \\
\gamma \text {-Alumina }\end{array}$ & $\begin{array}{l}\text { BASF } \\
\text { Thermal decomposition of } \\
\text { alum }\end{array}$ & 59.3 & {$[27]$} \\
$\begin{array}{l}\alpha \text {-Alumina } \\
\text { Zeolithe 5A }\end{array}$ & Calcination of $\gamma$-Alumina & 28 & {$[51]$} \\
$\begin{array}{l}\text { Activated } \\
\text { carbon }\end{array}$ & Alltech & 242.0 & {$[27]$} \\
$\begin{array}{l}\text { Ceria } \\
\text { Ceria }\end{array}$ & Norit & 230.3 & {$[27]$} \\
Ceria & $\quad 800^{\circ} \mathrm{C}$ calcined oxalate & 27.5 & {$[24]$} \\
\hline
\end{tabular}

new energy sites. The chemical surface does not alone explain the $\gamma_{s}^{\text {d }}$ difference. Another parameter which can influence the adsorption of the probe is the surface morphology. This latter can be affected by the oxide preparation.

A large panel of polar probes was tested to investigate polar interactions (ethanol, chloroform, acetonitrile, dichloromethane, dimethyl sulfoxide, acetone, ethyl acetate, diethyl ether, tetrahydrofuran, dioxane, diethylamine). Polar probes were not eluted from the surface at low coverage showing a high affinity with the surface. Thermodesorption at higher temperature was needed to desorb polar probes. This result indicates an irreversible adsorption of polar probes at low coverage. It demonstrates very strong polar interactions on its surface. Ceria is a well-known catalyst [49] and then one can expect strong interactions on surface. Polar probes develop additional non-dispersive interactions with the surface leading to very strong overall interactions on the surface. It also suggests a high reactivity of surface and the feature might explain the surface high reactivity toward adsorbed water.

Cyclic alkanes and branched alkanes were also tested to investigate surface roughness and topology. These probes were not eluted from the surface at $150{ }^{\circ} \mathrm{C}$ showing a similar behavior to polar probes. This result emphasizes once more the high reactivity of the surface.

\subsection{Effect of hydration investigated by inverse gas chromatography}

IGC at infinite dilution was used to monitor surface evolution upon hydration. Results obtained with hydrated samples are shown in Fig. 2. The figure shows results for samples calcined at $800{ }^{\circ} \mathrm{C}$ (hydrated_1) and $650^{\circ} \mathrm{C}$ (hydrated_2). In both case, the dispersive component of the surface energy increased with hydration. $\gamma_{\mathrm{s}}^{\mathrm{d}}$ increased from $184 \pm 2 \mathrm{~mJ} \mathrm{~m}^{-2}$ to $232 \pm 2 \mathrm{~mJ} \mathrm{~m}^{-2}$ for sample calcined at $800{ }^{\circ} \mathrm{C}$ and from $221 \pm 2 \mathrm{~mJ} \mathrm{~m}^{-2}$ to $240 \pm 2 \mathrm{~mJ} \mathrm{~m}^{-2}$ for sample calcined at $650^{\circ} \mathrm{C}$. These observations imply that storing the samples under controlled humid atmosphere induces an evolution of the surface. The effect is more noticeable on sample calcined at $800^{\circ} \mathrm{C}$. The surface seems more destabilized by hydration than in the case of the sample calcined at $650{ }^{\circ} \mathrm{C}$. The destabilization is in terms of interaction between oxide surface and molecular probes. After hydration, the molecular probes interact more strongly with the oxide surface. It shows that different calcination temperature induces different reactivity of the oxide surface. Increasing calcination temperature leads to stabilization of surface (decrease of $\gamma_{s}^{\mathrm{d}}$ ) (Fig. 2) and hydration has stronger impacts on high temperature calcined sample, i.e. more stable surface. Despite an apparent stabilization of the surface, high temperature calcination, (Fig. 2) leads to a higher reactivity toward hydration. One can propose that the evolution induced by hydration is explained by the opposite mechanism explaining the stabilization of the surface with increasing calcination temperature. The 

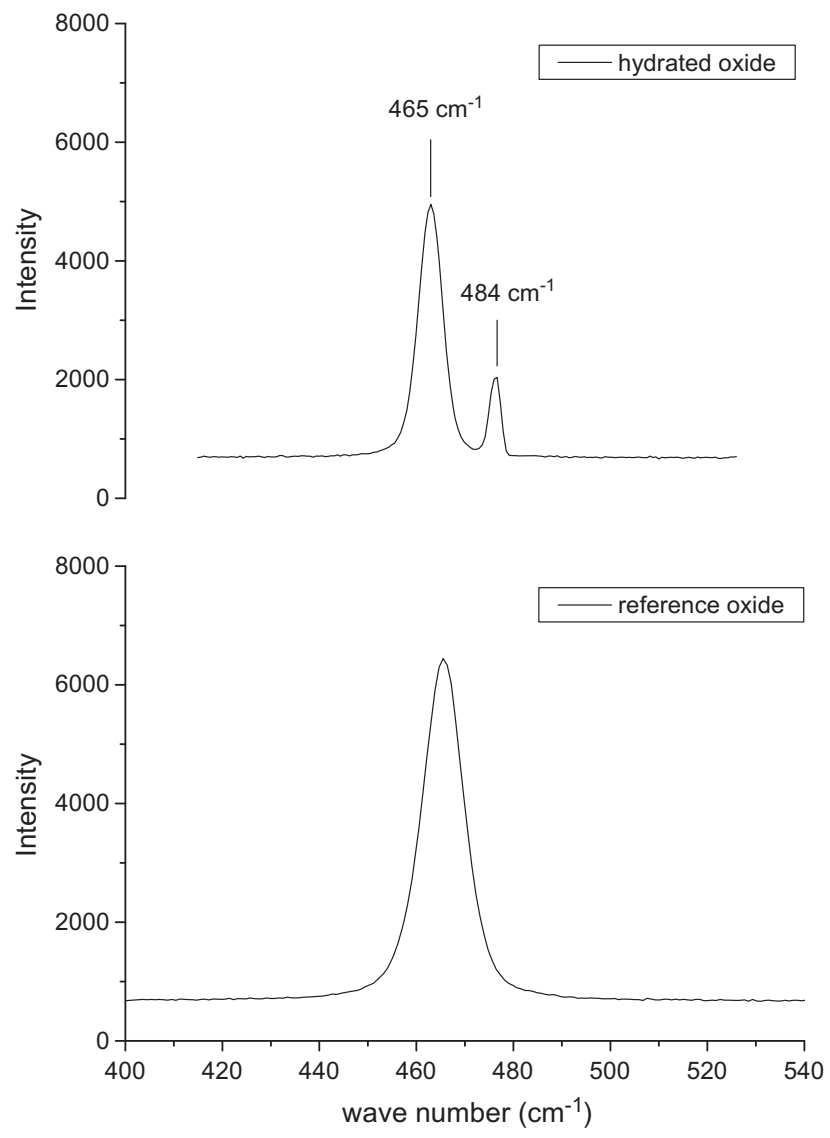

Fig. 3. Raman spectra of hydrated sample and reference sample (using in situ furnace).

increase of the $\gamma_{s}^{\mathrm{d}}$ value for the sample calcined at $650^{\circ} \mathrm{C}$ (Reference_2) is lower because the surface is already in a state closer to the hydrated one compared to the sample calcined at $800^{\circ} \mathrm{C}$ (Reference_1). The potential evolution is then lower. For the samples kept under humid atmosphere during 16 days, the dispersive component of the surface energy for the sample calcined at $800^{\circ} \mathrm{C}$ (hydrated_1) could come equal to the dispersive component for the sample calcined at $650{ }^{\circ} \mathrm{C}$ (hydrated_2). The evolution of the surface would have reached a final state corresponding to a highest value for the dispersive component of the surface energy. It has to be kept in mind that the measurements only reflect the behavior of the most energetic adsorption site. Nevertheless, these particular adsorption sites can reflect the evolution of the overall surface of the oxide. The measurement of the dispersive component only accounts for London interactions. The strong adsorption of polar probes suggests strong specific interactions. A precise description of the surface should include the specific component of the surface energy. However, the measurement of the dispersive component allows already to understand the evolution of the surface state with hydration and thermal treatment.

\subsection{Surface regeneration by thermal treatment}

The purpose was to investigate the ability to regenerate the surface to a reference state by thermal treatment. For the thermally treated samples, the Fig. 2 shows a decrease of the dispersive component of the surface energy in both case (high and low calcination temperature) induced by the thermal treatment. The decrease is $19 \mathrm{~mJ} \mathrm{~m}^{-2}$ and $6 \mathrm{~mJ} \mathrm{~m}^{-2}$ for treated_1 $\left(800^{\circ} \mathrm{C}\right)$ and treated-2 $\left(650^{\circ} \mathrm{C}\right.$ ) respectively (uncertainty $= \pm 2 \mathrm{~mJ} \mathrm{~m}^{-2}$ ). The tendency is

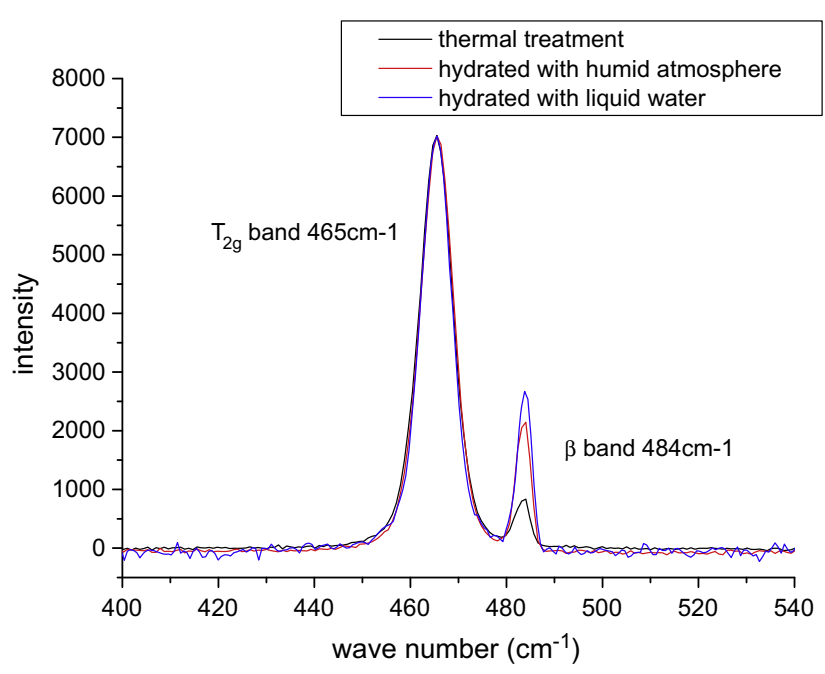

(A)

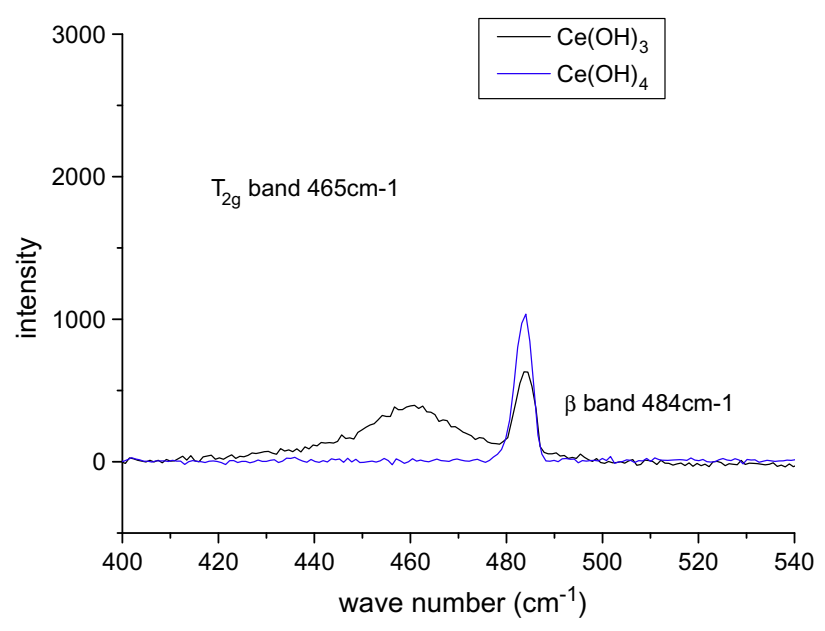

(B)

Fig. 4. (A) Raman spectra of sample with different preparations (thermal treatment $\left(800^{\circ} \mathrm{C} / \mathrm{Air}\right)$, humid atmosphere $(60 \%-6$ months), liquid water (overnight)) (normalized with $\mathrm{T}_{2 \mathrm{~g}}$ band), and (B) Raman spectra of cerium hydroxides (cerium(III) hydroxide, cerium (IV) hydroxide).

Table 5

$\beta / \mathrm{T}_{2 \mathrm{~g}}$ band area ratio for samples with different preparation.

\begin{tabular}{ll}
\hline & $\beta \mathrm{T}_{2 \mathrm{~g}}$ ratio \\
\hline Thermal treatment & 0.06 \\
Humid atmosphere & 0.15 \\
Water & 0.18 \\
\hline
\end{tabular}

the same for both samples although the sample calcined at $800{ }^{\circ} \mathrm{C}$ seems more affected by the thermal treatment. This result confirms that the calcination temperature is a crucial parameter for surface reactivity. Comparison with the initial state before hydration shows a gap of $29 \mathrm{~mJ} \mathrm{~m}^{-2}$ and $13 \mathrm{~mJ} \mathrm{~m}^{-2}$ for treated_1 $\left(800^{\circ} \mathrm{C}\right)$ and treated- $\left(650^{\circ} \mathrm{C}\right)$ respectively. The regeneration of the surface shows a higher impact on $800{ }^{\circ} \mathrm{C}$ calcined sample since the decrease of $\gamma_{s}^{\mathrm{d}}$ is higher. However, the final value is closer to the initial one for the $650{ }^{\circ} \mathrm{C}$ calcined sample. The calcination of oxalate at two different temperature leads to different surface states that behave differently upon hydration as shown by $\gamma_{s}^{\mathrm{d}}$ value. The surface states of the hydrated samples are then different and the 


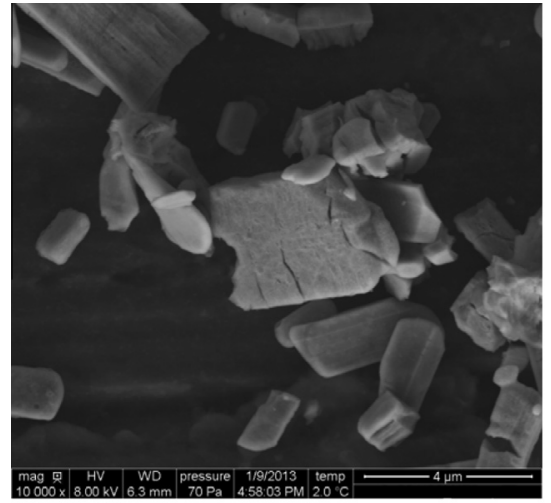

(A)

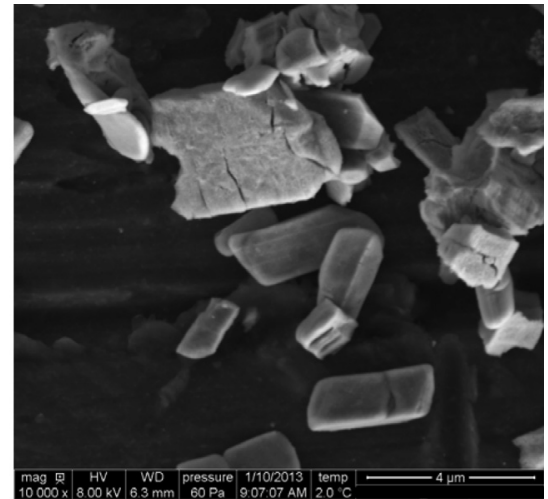

(B)

Fig. 5. In situ Hydration-ESEM micrographs of the ceria samples: (A) before in situ hydration, and (B) after in situ hydration.
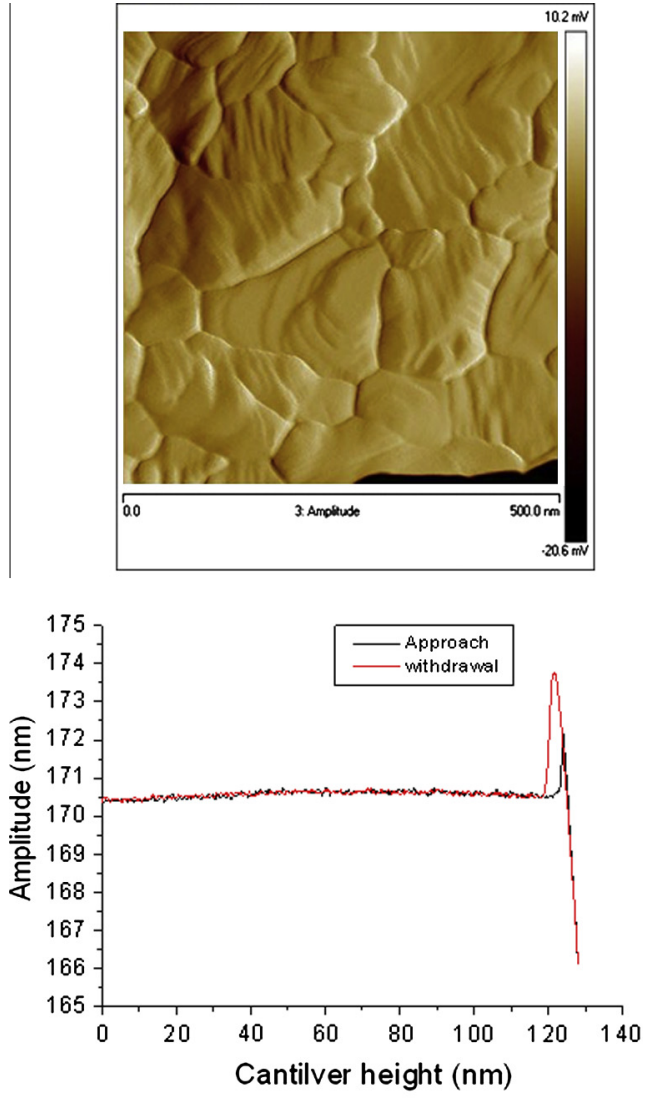

(A)
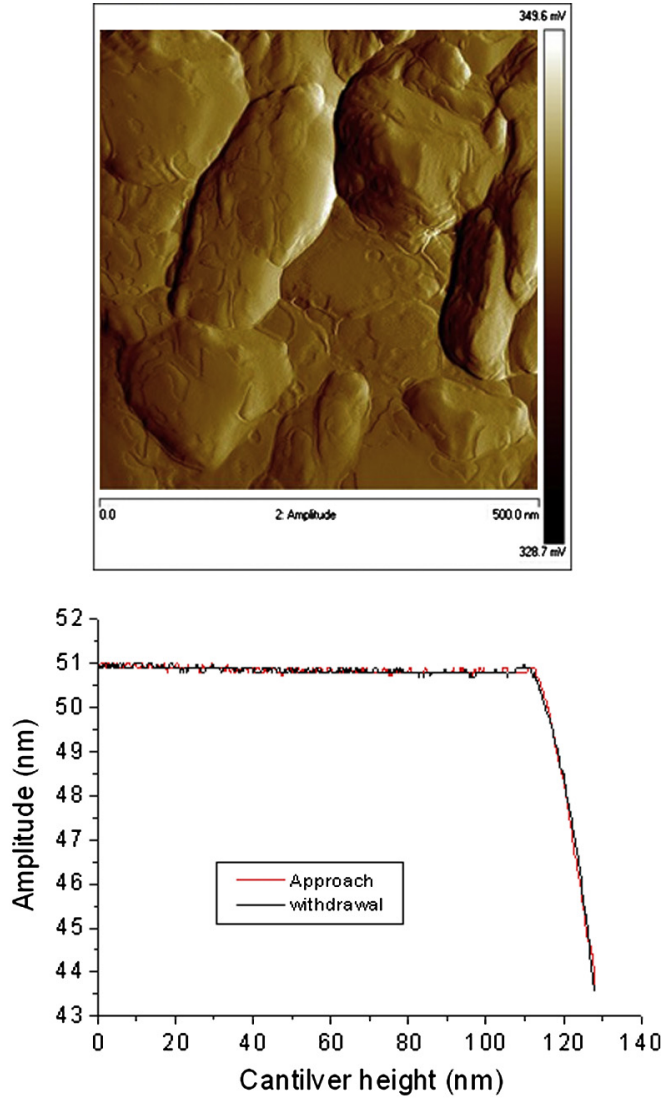

(B)

Fig. 6. Top: AFM amplitude images of the ceria samples and bottom: amplitude variation versus surface distance on (A) non-hydrated sample, and (B) sample hydrated with liquid water ex situ during 6 days.

surfaces have not the same ability for regeneration at $550^{\circ} \mathrm{C}$. This observation might be explained by the thermal treatment temperature. A higher temperature for thermal treatment might lead to a $\gamma_{s}^{\mathrm{d}}$ value for the $800^{\circ} \mathrm{C}$ calcined sample closer to the initial one. Furthermore, the $\gamma_{s}^{\mathrm{d}}$ values decrease with thermal treatment but are higher than values for the surface in the reference state (prehydration). The temperature of the thermal treatment might not be sufficiently high to induce a complete regeneration of the surface. The atmosphere during the thermal treatment is likewise a parameter that can influence the regeneration of the surface.

\subsection{Investigations using $\mu$-Raman spectroscopy}

Raman spectroscopy was used to investigate apparition of additional band induced by surface hydration beside $\mathrm{T}_{2 \mathrm{~g}}, 1 \mathrm{LO}$ and $2 \mathrm{LO}$ bands. Ceria sample was treated at $800{ }^{\circ} \mathrm{C}$ under static air for $2 \mathrm{~h}$ in in situ furnace. Sample was then cooled down to record spectrum at ambient temperature to avoid blackbody radiation disturbances. Recorded spectrum after in situ thermal treatment is presented in Fig. 3. Spectrum shows only one band at $465 \mathrm{~cm}^{-1}$ corresponding to $\mathrm{T}_{2 \mathrm{~g}}$ vibration band indicating a well ordered material. No addi- 
tional band is observed which suggests non-crystal disorders. This result is expected for a reference sample and is in good agreement with literature $[35,43,50]$. In situ furnace with high temperature ensured recording of a Raman spectrum representative of a nonhydrated surface.

Detailed spectra for hydrated sample are shown in Fig. 3. The spectrum for hydrated sample shows an additional band (named here $\beta$ band) beside the $T_{2 g}$ at $484 \mathrm{~cm}^{-1}$. Filters were used to provide low power laser beam $\left(6 \times 10^{-3} \mathrm{~mW}\right)$ to prevent any laser induced surface modification. IGC measurements show that thermal treatment is able to regenerate the surface. The local heating induced by laser irradiation can then annealed the surface alteration by hydration. Ceria is hydrated during 2 months under $80 \%$ relative humidity. No additional bands (1LO band, 2LO band) are observed at wave number higher than $1000 \mathrm{~cm}^{-1}$ for any sample. The presence of the $\beta$ band suggests the modification of the surface state.

To investigate the origin of this additional band, ceria was hydrated using liquid water to induce extreme conditions and enhanced surface evolution. Fig. 4 shows the recorded spectra. For the sample hydrated with water, the $\beta$ band is present and the band area ratio is higher than in the case of the samples hydrated by humid atmosphere (Table 5). This result suggests that the $\beta$ band is related to ceria reaction toward water since the use of liquid water leads to a modification of the Raman spectra of ceria.

The sample used for reference state (Fig. 3) was stored overnight under ambient conditions (normal conditions of temperature, pressure and humidity) and Raman spectrum was recorded the day after (Fig. 4). Different preparations lead to the same two bands: $T_{2 g}$ band at $465 \mathrm{~cm}^{-1}$ and the $\beta$ band at $484 \mathrm{~cm}^{-1}$. It appears that the band area ratios differ with the sample preparation. The thermally treated sample shows the lowest ratio and the liquid water hydrated sample the highest one. Samples hydrated by humid atmospheric atmosphere show intermediate values. This result is in good agreement with conclusions drawn from IGC-ID results on regeneration of the surface by thermal treatment. Thermal treatment is able to reduce the ratio of the areas of the two bands, implying the regeneration of the surface toward a reference state. Since the thermally treated sample is expected to be the less hydrated one, it confirms that the additional band is explained by the hydration of the surface. However, the sample is kept under atmospheric ambiance which contains water. The additional band might be due to a rapid rehydration of the sample overnight.

To investigate the nature of the surface modification, Raman spectra of cerium(III) and cerium(IV) hydroxides were recorded in the same conditions as previous oxide samples. (Fig. 4). The two samples show the same band at $484 \mathrm{~cm}^{-1}$. The spectra confirm the usual poor cristallinity of hydroxides. This band might be explained by the vibration of $\mathrm{Ce}-\mathrm{OH}$ bound on surface. This result suggests the formation of a thin hydroxide layer on the oxide surface. The oxidation state of cerium on the surface remains unknown since the two hydroxides spectra show the same feature at $484 \mathrm{~cm}^{-1}$. Spectrum of Ce(III) hydroxide shows a weak band around $460 \mathrm{~cm}^{-1}$. The origin of this band remains unknown.

\subsection{Evolution of surface topology: Environmental scanning electron microscopy (ESEM) and atomic force spectroscopy (AFM)}

ESEM enables to hydrate sample in situ and microstructure evolution can be followed on specific particles. Fig. 5 shows the surface evolution upon hydration of the exact same particle. Oxide sample is constituted of lamellar-shaped aggregates of micrometric size. This morphology is expected for oxide prepared by oxalate route [47]. In situ hydration was performed at 95\% relative humidity at pressure below $100 \mathrm{~Pa}$. No significant change is observed on micrographs. The sample was dried before experiment at $800^{\circ} \mathrm{C}$ under static air during $10 \mathrm{~min}$. ESEM experiments suggest that the sur- face evolution takes place at a smaller scale. To perform analysis at a smaller scale, AFM technique was used. This technique permits to observe surface topology down to the nanometer scale. A noncontact method has already been applied on ceria in ultra-high vacuum (UHV) conditions to study water adsorption on $\mathrm{CeO}_{2}$ (111) thin film [48]. UHV permits to limit water adsorption to submonolayer coverage. Authors were able to show adsorption of individual water molecule on specific sites. Fig. 6 shows AFM amplitude images of both reference surface and hydrated surfaces using tapping mode in air. Reference surface corresponds to ceria sample treated at $800^{\circ} \mathrm{C}$ before experiment. Hydration was obtained using deposition of liquid water for six days to enforce surface evolution. AFM images reveal an important evolution of the surface grain topology due to hydration. This observation was made on several different particles to check consistency. This result might be explained by the formation of a hydroxide superficial layer and confirms the nanometer scale of surface evolution. The measurement of amplitude variation versus distance was also recorded. Typical result is shown in Fig. 6. The measurement was reproduced on 35 different sites on both surfaces. The result appears very reproducible from one point to the other. The reference surface shows a strong interaction between the cantilever tip and surface during both approaching and retracting curves (peaks in the left curve). No particular interactions are suggested in the case of the hydrated sample. Tip-surface interaction measured by AFM force curves is completely different on non-hydrated and hydrated surfaces.

\section{Conclusions}

Ceria surface evolution upon hydration is studied using different techniques. Characterization of the reference surface state indicates a high complexity of ceria surface when prepared by oxalate calcination which is similar to plutonium oxide preparation. The finite solute concentration method allows to determine the distribution of adsorption sites. Ceria surface shows three different types of adsorption sites for isopropanol. Inverse gas chromatography at infinite dilution gives a good insight of the surface reactivity toward water. The calcination temperature influences the surface reactivity regarding hydration. A thermal treatment at moderate temperature seems to regenerate at least partially the initial feature of the surface. However, a higher temperature for thermal treatment could allow to induce a complete regeneration of the surface. $\mu$-Raman spectroscopy confirms the modification of the surface with the emergence of an additional band close to $T_{2 g}$ vibration band upon surface hydration suggesting hydroxide superficial layer. Raman spectroscopy experiments confirm IGC results: thermal treatment leads to a regeneration of the surface. ESEM and AFM show that the surface modification takes place at a nanometer scale and AFM results suggest the formation of superficial layer with an important evolution of surface nanostructure. $\mathrm{PuO}_{2}$ surface is expected to follow a close behavior to $\mathrm{CeO}_{2}$ surface. The surface evolution upon hydration is expected to influence the radiation chemistry occurring at $\mathrm{PuO}_{2}$ surface. Further experiments are expected to better understand the modification during thermal treatment and to estimate the kinetics of surface hydration and, in the case of plutonium dioxide, to relate the generation of $\mathrm{H}_{2}$ due to radiolysis of adsorbed water to the surface modification upon adsorption.

\section{Supporting Information}

Determination of suitable probe amount in IGC-FC, Determination of desorption isotherm from IGC-FC chromatographic peak, IGC-ID chromatographic peak and examples of n-alcanes lines. This 
material is available free of charge via the Internet at http://pubs.acs.org/.

\section{Acknowledgment}

The authors would like to thank AREVA NC for financial support and CEA future fuel cycle back-end objectives management.

\section{References}

[1] V. Onufriev, Status and Advances in MOX Fuel Technology, IAEA, 2003.

[2] J.M. Haschke, T.E. Ricketts, Plutonium Dioxide Storage: Conditions for Preparation and Handling, Las Alamos National Laboratory, 1995.

[3] J.L. Stakebake, L.M. Steward, J. Colloid Interface Sci. 42 (1973) 328-333.

[4] A. Benhamou, J.P. Beraud, Analusis 8 (1980) 376-380.

[5] M.V. Vladimirova, I.A. Kulikov, Radiochemistry 44 (2001) 86-90.

[6] J.M. Duffey, R.R. Livinston, Gas Generation Testing of Plutonium Dioxide, Westinghouse Savannah River Company, 2002.

[7] J.M. Duffey et al., Pressure Development in Sealed Containers with PlutoniumBearing Materials, Savannah River National Laboratory, 2010.

[8] P.M. Almond et al., Gas Analyses From Headspace of Plutonium-Bearing Materials Containers, Savannah River National Laboratory, 2010.

[9] H.E. Sims et al., J. Nucl. Mater 437 (2013) 359-364.

[10] J.L. Stakebake et al., J. Alloys Compd. 202 (1993) 251-263.

[11] J.M. Haschke, T.H. Allen, Interactions of Plutonium Dioxide with Water and Oxygen-Hydrogen Mixtures, Las Alamos National Laboratory, 1999.

[12] S.D. Conradson et al., Inorg. Chem. 42 (2003) 3715-3717.

[13] J.D. Farr et al., J. Nucl. Mater. 328 (2004) 124-136.

[14] T. Gouder et al., J. Surf. Sci. 601 (2007) L77-L80.

[15] A. Seibert et al., Radiochim. Acta 98 (2010) 647-657.

[16] C. Madic, Science 287 (2000) 243-244.

[17] P.A. Thiel, T.E. Madey, Surf. Sci. Reports 7 (1987) 211-385.

[18] M.A. Henderson, Surf. Sci. Reports 46 (2002) 1-308.
[19] M. Molinari et al., J. Phys. Chem. C 116 (2012) 7073-7082.

[20] S. Hayun et al., J. Am. Ceram. Soc. 94 (2011) 3992-3999.

[21] D. Fernandez-Torre et al., J. Phys. Chem. C 116 (2012) 13584-13593.

[22] E. Brendle et al., J. Chim. Phys. Phys.-Chim. Biol. 95 (1998) 1685-1710.

[23] Y. Al-saigh Zeki, Int. J. Polym. Anal. Charact. 3 (1997) 249-291.

[24] E. Díaz et al., J. Chromatogr. A 1116 (2006) 230-239.

[25] E. Papirer et al., J. Mater. Sci. 35 (2000) 3573-3577.

[26] L. Boudriche et al., Colloids Surf. - Physicochem. Eng. Asp. 399 (2012) 1-10.

[27] E. Diaz et al., J. Chromatogr. A 1049 (2004) 139-146.

[28] C. Jégou et al., J. Nucl. Mater. 405 (2010) 235-243.

[29] C. Jégou et al., J. Nucl. Mater. 399 (2010) 68-80.

[30] V.G. Keramidas, J. Chem. Phys. 59 (1973) 1561.

[31] G.M. Begun et al., J. Less Common Met. 162 (1990) 129-133.

[32] J. Schoenes, J. Phys. Reports 63 (1980) 301-336.

[33] D. Manara, B. Renker, J. Nucl. Mater. 321 (2003) 233-237.

[34] J.M. Sarsfield et al., J. Nucl. Mater. 427 (2012) 333-342.

[35] W. Weber et al., J. Phys. Rev. B 48 (1993) 178-185.

[36] G. Binnig et al., Phys. Rev. Lett. 56 (1986) 930-933.

[37] J.V. Lauritsen, M. Reichling, J. Phys. Condens. Matter 22 (2010) 263001.

[38] S. Gritschneder et al., Nanotechnology 18 (2007) 044025.

[39] J.C. Marra et al., Cerium as a Surrogate in the Plutonium Immobilized Form, American Chemical Society, Washington, DC, 2006.

[40] X. Machuron-Mandard, C. Madic, J. Alloys Compd. 235 (1996) 216-224.

[41] L. Claparede et al., Inorg. Chem. 50 (2011) 11702-11714.

[42] A. Badri et al., J. Chem. Soc. Faraday Trans. 92 (1996) 4669.

[43] D. Horlait et al., Inorg. Chem. 50 (2011) 7150-7161.

[44] J.R. Conder, C.L. Young, Physicochemical Measurement by Gas Chromatography, Wiley, 1979.

[45] H. Balard et al., Langmuir 13 (1997) 1251-1255.

[46] G.M. Dorris, D.G. Gray, J. Colloid Interface Sci. 77 (1980) 353-362.

[47] L. Claparede et al., Inorg. Chem. 50 (2011) 9059-9072.

[48] J.Y. Heng et al., Pharm. Res. 23 (2006) 1918-1927.

[49] A. Trovarelli, Catal. Rev. Sci. Eng 38 (1996) 439-520.

[50] C.T. Nottbohm, C. Hess, Catal. Commun. 22 (2012) 39-42.

[51] C. Tisserand et al., Powder Technol. 190 (2009) 53-58. 